PNNL-19073

\title{
Implementation Plans for a Systems Microbiology and Extremophile Research Facility
}

Summary of a workshop held March $26^{\text {th }}, 2008$ Environmental Molecular Sciences Laboratory Richland, WA

April 2009

Prepared for the U.S. Department of Energy's Office of Biological and Environmental Research under Contract DE-AC0576RL01830

Pacific Northwest National Laboratory Richland, Washington 99352 


\title{
DISCLAIMER
}

This report was prepared as an account of work sponsored by an agency of the United States Government. Neither the United States Government nor any agency thereof, nor Battelle Memorial Institute, nor any of their employees, makes any warranty, express or implied, or assumes any legal liability or responsibility for the accuracy, completeness, or usefulness of any information, apparatus, product, or process disclosed, or represents that its use would not infringe privately owned rights. Reference herein to any specific commercial product, process, or service by trade name, trademark, manufacturer, or otherwise does not necessarily constitute or imply its endorsement, recommendation, or favoring by the United States Government or any agency thereof, or Battelle Memorial Institute. The views and opinions of authors expressed herein do not necessarily state or reflect those of the United States Government or any agency thereof.

\author{
PACIFIC NORTHWEST NATIONAL LABORATORY \\ operated by \\ BATTELLE \\ for the \\ UNITED STATES DEPARTMENT OF ENERGY \\ under Contract DE-AC05-76RL01830
}

Printed in the United States of America

Available to DOE and DOE contractors from the

Office of Scientific and Technical Information,

P.O. Box 62, Oak Ridge, TN 37831-0062;

ph: (865) 576-8401

fax: $(865) 576-5728$

email: reports@adonis.osti.gov

\footnotetext{
Available to the public from the National Technical Information Service, U.S. Department of Commerce, 5285 Port Royal Rd., Springfield, VA 22161 ph: (800) 553-6847 fax: $(703) 605-6900$

email: orders@ntis.fedworld.gov

online ordering: http://www.ntis.gov/ordering.htm
}

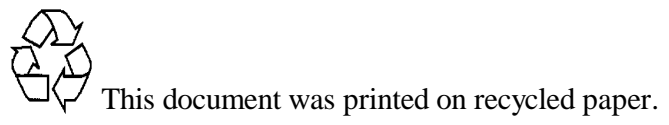




\section{Implementation Plans for a Systems Microbiology and Extremophile Research Facility}

Summary of a workshop held March 26th, 2008

Environmental Molecular Sciences Laboratory

Richland, WA

HS Wiley

April 2009

Prepared for the U.S. Department of Energy's Office of Biological and Environmental Research under Contract DE-AC05-76RL01830

Pacific Northwest National Laboratory

Richland, Washington 99352 


\section{Introduction}

Biological organisms long ago "solved" many problems for which scientists and engineers seek solutions. Microbes in particular offer an astonishingly diverse set of capabilities that can help revolutionize our approach to solving many important DOE problems. For example, photosynthetic organisms can generate hydrogen from light while simultaneously sequestering carbon. Others can produce enzymes that break down cellulose and other biomass to produce liquid fuels. Microbes in water and soil can capture carbon and store it in the earth and ocean depths. Understanding the dynamic interaction between living organisms and the environment is critical to predicting and mitigating the impacts of energy-production-related activities on the environment and human health.

Collectively, microorganisms contain most of the biochemical diversity on Earth and they comprise nearly one-half of its biomass. They primary impact the planet by acting as catalysts of biogeochemical cycles; they capture light energy and fix $\mathrm{CO}_{2}$ in the worlds oceans, they degrade plant polymers and convert them to humus in soils, they weather rocks and facilitate mineral precipitation. Although the ability of selected microorganisms to participate in these processes is known, they rarely live in monoculture but rather function within communities. In spite of this, little is known about the composition of microbial communities and how individual species function within them. We lack an understanding of the nature of the individual organisms and their genes, how they interact to perform complex functions such as energy and materials exchange, how they sense and respond to their environment and how they evolve and adapt to environmental change. Understanding these aspects of microbes and their communities would be transformational with far-reaching impacts on climate, energy and human health. This knowledge would create a foundation for predicting their behavior and, ultimately, manipulating them to solve DOE problems.

Recent advances in whole-genome sequencing for a variety of organisms and improvements in high-throughput instrumentation have contributed to a rapid transition of the biological research paradigm towards understanding biology at a systems level. As a result, biology is evolving from a descriptive to a quantitative, ultimately predictive science where the ability to collect and productively use large amounts of biological data is crucial. Understanding how the ensemble of proteins in cells gives rise to biological outcomes is fundamental to systems biology. These advances will require new technologies and approaches to measure and track the temporal and spatial disposition of proteins in cells and how networks of proteins and other regulatory molecules give rise to specific activities.

The DOE has a strong interest in promoting the application of systems biology to understanding microbial function and this comprises a major focus of its Genomics:GTL program. A major problem in pursuing what has been termed "systems microbiology" is the lack of the facilities and infrastructure for conducting this new style of research. To solve this problem, the Genomics:GTL program has funded a number of large-scale research centers focused on either mission-oriented outcomes, such as bioenergy, or basic technologies, such as gene sequencing, high-throughput proteomics or the identification of protein complexes. Although these centers generate data that will be useful to the research community, their scientific goals are relatively narrow and are not designed to accommodate the general community need for advanced capabilities for systems microbiology research. 


\section{Workshop background}

The original idea of creating the capabilities at EMSL to drive research in systems microbiology was an outcome of the workshop on "The Development of New User Research Capabilities in Environmental Molecular Science”, which was held August 1-2, 2006. This meeting included 104 expert scientists-many representing EMSL users-from 40 institutions, including 24 universities and 5 national laboratories. Discussions during this meeting centered around future science and technology challenges and the capabilities that these scientists would need in the future to further their research.

A topic of very active discussion at this workshop was providing advanced research capabilities in the general area of microbial dynamics and visualization. After obtaining feedback on the initial workshop report and consulting with potential users in this area of research, we refined the concept of creating unique capabilities within EMSL that would better support the needs of the microbiology community and be relevant to DOE mission areas. Our refined goals include the building of integrated research capabilities in the area of "systems microbiology", with an explicit inclusion of extremophiles. Extremophiles are an extremely interesting group of microorganisms that can withstand extremes of temperature, $\mathrm{pH}$ and ionic strength, which are conditions that are frequently found at field sites of interest to DOE. It is important to not only understand how these extreme conditions impact microorganisms, but also how to take advantage of those microbes that are tolerant to them. For example, enzymes derived from extremophiles can function under harsh conditions, making them particularly suitable for industrial applications. However, extremophiles are difficult to culture under standard laboratory conditions, and many are thus poorly characterized. There was a general consensus at the workshop that a facility to grow extremophiles and other microbial communities under well-controlled and regulated conditions could be extremely useful to a large group of microbiologists. The additional ability to generate and integrate high-throughput molecular data with physiological response measurements could revolutionize research in this area.

It became clear that it would be highly desirable to build an integrated capability for growing and analyzing both extremophiles and microbial communities. This would require implementing corrosion-resistant systems for growing microbes under conditions of elevated ionic strength and temperature, parallel culture systems and instruments for the growth of microbial biofilms. These systems should allow continuous monitoring, sampling, and analysis of the microbial cultures. Because many environmentally relevant microbes grow slowly, particularly extremophiles, the culture facilities should support remote monitoring and allow different analyses to be run in response to computer commands. This would allow users to set up a culture experiment on-site, but still be able to monitor and control the running experiment from their home institution. These culture facilities would be complemented by a new set of cutting-edge imaging technologies that would allow the visualization of metabolites and gene expression profiles in living cells. These capabilities would create extremely rich datasets for understanding metabolic and gene regulatory networks. This would provide a fundamental basis for understanding microbial functions at a systems level, which is necessary for future efforts in re-engineering microbial systems for a variety of different uses.

We also became aware that the technical challenges that face biology in its transition to a predictive science have been the subject of many different workshops and scientific meetings over the last several years. We used the reports from these workshops in addition to the information gathered from EMSL users to create an initial blueprint for the new biology capabilities at EMSL. The most important reports that we have used are: 
American Academy of Microbiology workshop "Systems Microbiology: Beyond Microbial

Genomics," December 2004 (http://www.asm.org/academy/index.asp?bid=32329)

WTEC report “Assessment of International Research and Development in Systems Biology”. Final workshop held at NSF March 2005. Proceedings published by Springer in Hardcover: 262 pages, 2007 (http://www.wtec.org/sysbio)

US-EC workshop on "Infrastructure needs of systems biology”, May 2007

(http://ec.europa.eu/research/biotechnology/ec-us/docs/us_ec_syst_biology_workshop.pdf)

DOE Genomics:GTL roadmap “Systems Biology for Energy and Environment,” August 2005 (http://genomicsgtl.energy.gov/roadmap/index.shtml)
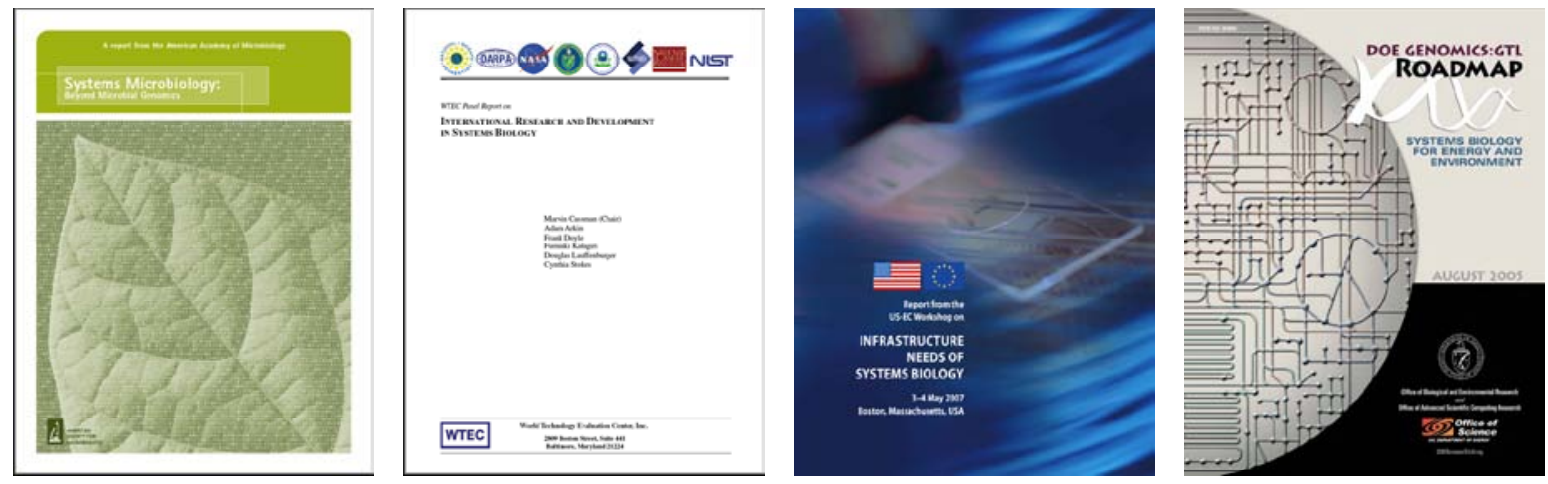

These workshops have explored the current state-of-the-art in systems biology and the challenges that are being faced by the biological community during the transition to systems biology. Of particular importance has been the identification of technologies and capabilities that would significantly advance progress of this area of research. Those needs that fit within the potential technical capabilities of the EMSL over the next decade were identified as future research areas to explore.

One area of particular concern was how biologists were expected to interact with a facility that generated high-throughput data. Most biologists have limited experience in either generating or interpreting this type of data or in analyzing their model organism at a more "systems level". If the facility is expected to become a resource that is useful to a variety of different investigators, then defining its operational logistics will be a very important part of the design process. This was a major conclusion of the Workshop on Experimental Access Portals for a Global Proteomics Facility, sponsored by PNNL and held at Gaithersburg, Maryland, June 2-3, 2003. The workshop's primary purpose was to define ways in which a community of scientists potentially could interact with large core facilities. The findings and recommendations of this workshop (excerpted in Appendix B) provide a useful starting point for examining the issue of how largescale resources can be used most effectively to drive microbial research.

\section{Initial Concepts}

The information gathered from the workshops and reports descried above were used to create an initial concept for a "Systems Microbiology and Extremophile Research Facility (SMERF)". This facility was specifically designed to allow microbiologists to conduct cutting-edge, systemsoriented research within EMSL. The basic concept of the SMERF was a set of capabilities for the controlled cultivation of a wide variety of different microorganisms together with an integrated data management systems that allowed the high-throughput data generated within the EMSL to 
be used to understand the molecular mechanisms that underlie specific cellular functions. Additional analytical instrumentation, particularly in the area of live cell imaging would complement current EMSL capabilities in proteomics, metabolomics and structural biology.

The equipment for the cultivation and analysis of microbes was originally envisioned to include:

1) Chemostat-based instrumentation for analysis of cells grown under rigorously defined conditions, including extreme environmental conditions.

2) Parallel culture systems to analyze cellular composition and responses under many different conditions, or to analyze different strains under the same conditions.

3) Biofilm-based instrumentation to understand interactions between cells in a community as well as their interactions with solid surfaces.

4) High performance imaging systems (respect to both resolution and speed) that can follow the distribution of proteins within a cell or the pattern of gene expression in a population of cells.

Much of the proposed instrumentation for SMERF was initially conceived to be used for continual live-cell monitoring of either the chemostats or biofilms growth chambers, but instrumentation for fractionating and analyzing externally provided samples was also thought to be needed. Monitoring would include both molecular and cell-based measurements.

For the chemostat-based instrumentation, the initial concept was a collection of bioreactors that would be modified to allow continuous sampling. Cell samples could be analyzed immediately using attached flow cytometers or microscope flow cells. Alternately, samples could be automatically concentrated and flash-frozen for later biochemical analysis (e.g., proteomic, genomic, and metabolite analysis). Headspace gas samples could also be continuously sampled and analyzed by mass spectrometry and the medium could be analyzed by HPLC for metabolite production or depletion. Data and samples would be collected and logged automatically by an integrated data management system. Chemostats could also be combined with NMR instrumentation to allow continuous monitoring of cellular metabolism. Bioreactors with controlled lighting would also be available for growth of photosynthetic organisms.

A central initial concept for the SMERF was that the primary role of the facility should be to generate controlled biological samples and the associated data. Multiple types of data are generally needed to understand complex cellular processes and these data are usually gathered under a variety of different conditions. However, the cellular machinery responsible for biological functions is generally sensitive to cellular context. Unless all biological samples are collected under identical conditions (or simultaneously), it will be very difficult to interpret the resulting data. Corollaries to this concept are that one must be able to grow the organisms under relevant conditions, be able to measure these conditions accurately and to relate the conditions to the biological data in a meaningful way. To accomplish this, a sophisticated data collection, management and integration infrastructure is required.

\section{Workshop purpose and Organization}

Once an initial concept for SMERF was created, it was necessary to vet these ideas to a group of users (and potential users) in this area of research. A list of potential workshop participants was solicited from Jim Fredrickson, Allan Konopka and members of the EMSL Scientific Advisory Committee. Participants were also selected based on their participation in similar workshops and their scientific prominence in the field of Systems Microbiology.

A total of 29 investigators attended the workshop on March 26 $6^{\text {th }}, 2008$ at the EMSL. This number included 13 scientists from outside of PNNL. Additional advisors who could not attend, but 
provided feedback on the workshop report included Anna-Louise Reysenbach, of Portland State; Edward DeLong of MIT and David Stahl at the University of Washington.

The workshop was scheduled for a single day. The first part of the morning was spent in a general session discussing the underlying concept of the SMERF. Himadri Pakrasi from Washington University, Nitin Baliga from the Institute of Systems Biology, and Steven Wiley and Allan Konopka from PNNL provided background talks on systems microbiology and some successful examples of the use of multidimensional data to understand complex microbial processes. The rest of the morning was spent discussing the feasibility and desirability of creating a systems microbiology and extremophile research facility in the EMSL. The afternoon was spent discussing specific implementation plans and refining concepts for the facility.

\section{Findings and Recommendations}

Recommendations made by the SMERF Workshop participants are summarized in brief immediately below. Details from the workshop discussion follow in Appendix A.

\section{General}

There was general enthusiasm for the concept of SMERF and broad agreement regarding its potential usefulness and impact on science. Its greatest value was felt to be in its potential to make the diverse capabilities of EMSL more accessible to the biological community. There was general interest in the concept of integrating multidimensional data sets for understanding complex biological processes, but many participants felt that the data management expertise for accomplishing this is not commonly available. If the idea of SMERF is to drive progress in systems microbiology, then targeted user training will be a necessary implementation detail. To be relevant to much of the environmental microbiology research that is being pursued by the scientific community, SMERF must be able to recreate experimental conditions that are relevant to specific field sites and be able to grow difficult organisms. Tight integration of the sample generation process in SMERF and the analytical capabilities of EMSL would provide great benefit to the user community.

SMERF should be designed to complement research projects being conducted as part of EMSL's Science Themes, in particular, the Biological Interactions and Dynamics and Geochemistry/Biogeochemistry and Subsurface Science Themes.

SMERF should focus on enabling research relevant to DOE missions. Microbes to be targeted for investigation should be connected to bioenergy/biofuels, carbon sequestration and management, bioremediation, and global warming research. This does not mean that other organisms cannot or should not be studied, but that the specific capabilities built into SMERF should have DOErelevant organisms as the target.

SMERF should be configured to specifically serve the needs of a scientific user facility. Workshop participants recommended that the design of SMERF should keep the challenges of a national scientific user facility in mind. A user facility:

- Must be responsive to the needs of the user community

- Must provide unique capabilities

- Needs to integrate multiple capabilities to support the vision of EMSL

- Should have general-purpose research laboratory space as part of the facility.

- Needs to be able to implement research techniques that are beyond those available at most university research facilities.

- Should be designed around solving particular classes of biological problems. 
Capabilities that should be part of SMERF

There was a great deal of enthusiasm for building culture capabilities for difficult-to-grow organisms and for generating sufficient biomass for a variety of different analyses, including protein purification and characterization. In particular, automated sampling methods that preserve the state of aerobic and anaerobic cells and, if necessary, accommodate rapid freezing were felt to be highly desirable.

\section{Growth and monitoring}

It was recommended to design and build remotely controlled parallel culture systems that can accommodate multiple users simultaneously. Because the amount of biomass required for a particular analysis can vary considerably, it was recommended to consider small-scale culture systems using microfluidics as well as larger scale chemostats. All culture systems should be able to accommodate multiple conditions and support automated sampling and remote monitoring if needed. Conditions of interest include high salt, high temperature, high pressure, high acidity, radioactivity, and controlled exposure to metal ions. Specific sets of environmental conditions should be able to be replicated as well, including different atmospheric conditions, soil habitats, high altitude, methane environments, and oil field conditions. Photosynthetic microbes should be accommodated such that light parameters and gas exchange are uniform and can be monitored and controlled.

The cell culture capability should use as many off-the-shelf components as possible, although system customization and integration will likely be required. Current commercial systems do not have the requisite high-throughput, multi-measurement capabilities envisioned for the SMERF, but the engineering teams at EMSL should be able to create suitable systems from those documented in the literature and in consultation with potential users of the facility. It is important that the configuration of the major culture system be flexible and modifiable for individual experiments. The culture systems should be usable for time-course analysis of metabolism (both metabolite utilization and generation), batch or continuous mode experiments, and permit ad hoc cell sampling for analysis of gene and protein expression patterns.

Dedicated culture systems should also be set up for different types of experiments. Long-term growth experiments will likely require chemostats whereas production of large amounts of biomass from extremophiles will require batch culture systems. Systems should also be available for the optimization of culture conditions for difficult-to-grow organisms, although the normal expectation is that growth conditions for a particular experimental organism should be standardized prior to initiating an experimental study within the SMERF.

The growth facility should be able to accommodate pure cultures, biofilms, and microbial communities. Biofilm-specific capabilities should complement, but not replicate, the existing facility at Montana State University. The ability to grow algae and plants should be explored in the context of specific research projects

An necessary capability is high-throughput phenotypic screening, where the response of a particular strain of microbe to specific environmental parameters can be monitored. This could done using a Biolog (http://www.biolog.com/pmTechDesOver.html) or Bioscreen instrument (http://www.growthcurvesusa.com/description.html). This capability would allow the response profile of a particular microbe to be comprehensively evaluated or differences in the profile of two different strains to be compared.

Analysis

EMSL already has many high-throughput analysis tools available for systems microbiology, but 
additional technologies would greatly enhance its attractiveness as a user facility for the biological community. Currently available capabilities relevant to the SMERF include global proteomics, the analysis of post-translational modification of proteins and combined NMRconfocal imaging of biofilms. Additional capabilities that are needed include image-based tools for mapping the spatial physiology of individual cells, communities, and biofilms as well as capabilities for isolating cells, such as flow cytometry and laser-capture microdissection.

High-throughput transcriptome analysis in the SMERF would be an extremely powerful addition to EMSL capabilities, especially with respect to functional profiling of microbial communities. Next generation sequencing technologies are now providing the means to quantify cellular RNA profiles without the need for specific probes or chips. This theoretically provides the ability to establish the composition as well as the gene expression profile of a complex microbial community. In combination with high-throughput proteomics, this promises to provide important new insights into community functions. The multiplexing ability of the new sequencing platforms also provides the ability to do massively parallel gene expression analysis, which should greatly assist in reconstructing the gene regulatory network of a specific microorganism.

Enhanced metabolomic capabilities are also needed to complement the currently limited capabilities in EMSL, including the ability to identify unknown metabolites, quantify certain sets of specific metabolites and measure the flux of metabolites through isotopic labeling methods. An emphasis should be placed on targeted metabolomics (also called metabolic profiling or the identification of signature metabolites).

For network and community studies, analytical techniques with high spatial resolution are important. Imaging technologies/capabilities that would move microbiology studies forward include fluorescence in-situ hybridization (FISH), imaging of microbes interacting with mineral surfaces and high-speed, multi-spectral imaging. Other technologies that should be pursued include microspectroscopy, Raman imaging and potentially imaging mass spectroscopy. Of high importance is the proposed Stochastic Optical Reconstruction Microscopy (STORM) imaging because no facility currently has the technology needed to examine cells below the optical limit of resolution. This capability would be in very high demand by biological users.

\section{Data management and integration}

It is essential for the SMERF to have a robust data management and analysis infrastructure. Many biologists are not conversant on how to manage and analyze large data sets and the ability of the SMERF to handle these tasks will be critical to its success in engaging the user community. Critical elements of data management and integration that must be addressed include metadata collection and dataset storage and providing user-friendly access to the large sets of data generated by EMSL. It is essential to have consultants available that can advise users regarding data integration and interpretation. It would also be highly useful to develop new tools for data integration and analysis and to utilize the EMSL computational resources to accelerate the performance of network inference algorithms. It will also be necessary to develop a policy on how the data gathered using EMSL resources would be made available to the research community, particularly with respect to data gathered on model organisms.

As a first step, a system should be created to manage data from projects that are being actively conducted in the SMERF. This system should link to the proteomics data management system in EMSL (PRISM) and well as allow management and retrieval of gene expression and imaging data generated from SMERF instruments. This system should allow both export of the data to standard formats as well as support data integration software, such as the Bioinformatics Resource Manager (BRM). 


\section{Prioritization and Implementation}

The time for implementation of the SMERF will necessarily be constrained by budgets and limitation in current science and technology. However, it is essential that implementation be pursued as rapidly as possible so that the scientific opportunities represented by the SMERF are not lost. The first goal should be to assemble sufficient capabilities and resources to allow the implementation of at least one pilot project that will drive the specification and integration of the capabilities that constitute the vision of SMERF. The principal requirement of this pilot project is that it be driven by a specific and important scientific question that would be extremely difficult to address without the integrated research capabilities of SMERF. This question should ideally be relevant to the DOE mission area.

Implementation should be staged to be maximally efficient. Engagement with potential users, funding mechanisms and logistics for identification and engagement of staff to support EMSL in this area are likely to be significant planning issues. It is recommended to utilize the Science Theme Advisory Panel (STAP) that is being created for the Biological Interactions and Dynamics science themes area in EMSL. The STAP is an advisory committee of potential users from both within PNNL and a representative from the EMSL Scientific Advisory Committee. Their role should include advising EMSL on appropriate pilot projects that should be used to launch the SMERF based on the importance of the scientific question, the relevance to DOE mission areas and its scientific and technical impact. The Advisory committee should also provide feedback on the technical capabilities needed for currently planed projects and those that will enable new classes of research projects. The committee could also provide assistance on the evaluation of proposals to utilize SMERF.

\section{Participants}

March 26 ${ }^{\text {th }}$, 2008, Richland, WA

Nitin Baliga

Institute of Systems Biology

nbaliga@systemsbiology.org

Brian Bothner

Montana State University

bbothner@chemistry.montana.edu

Allison Campbell

EMSL

Pacific Northwest National Laboratory

Alice Dohnalkova

Pacific Northwest National Laboratory

Andy Felmy

EMSL

Pacific Northwest National Laboratory

Matthew Fields

Montana State University

matthew.fields@erc.montana.edu
Jean Futrell

Pacific Northwest National Laboratory

Jil T. Geller

Lawrence Berkeley National Laboratory

JTGeller@lbl.gov

Roy Gephart

EMSL

Pacific Northwest National Laboratory

E. Peter Greenberg

University of Washington

epgreen@u.washington.edu

Nancy Hess

EMSL

Pacific Northwest National Laboratory

Patricia A. Holden

University of California Santa Barbara

holden@bren.ucsb.edu 
Dave Hoyt

EMSL

Pacific Northwest National Laboratory

Allan Konopka

Pacific Northwest National Laboratory

Dave Koppenaal

EMSL

Pacific Northwest National Laboratory

Mary E. Lidstrom

University of Washington

lidstrom@u.washington.edu

Jason McDermott

Pacific Northwest National Laboratory

Ellyn Murphy

Pacific Northwest National Laboratory

Galya Orr

Pacific Northwest National Laboratory

Himadri Pakrasi

Washington University, Saint Louis

Pakrasi@biology2.wustl.edu

Bernhard Palsson

University of California San Diego

palsson@ucsd.edu
Ljiljana Pasa-Tolic

EMSL

Pacific Northwest National Laboratory

Kevin Regimbal

EMSL

Pacific Northwest National Laboratory

Thomas M. Schmidt

Michigan State University

tschmidt@msu.edu

Dan Sisk

EMSL

Pacific Northwest National Laboratory

Ines Thiele

University of California San Diego

ines.thiele@gmail.com

Ger Van der Engh

Cytopeia Incorporated

engh@systemsbiology.org

David M Ward

Montana State University

umbdw@montana.edu

H Steven Wiley

EMSL

Pacific Northwest National Laboratory steven.wiley@pnl.gov 


\section{Appendix A: Workshop Discussion Details}

\section{Overview and discussions}

The meeting opened with talks from key speakers, including three experts in microbial research:

- Nitin S. Baliga from the Institute for Systems Biology

- Himadri Pakrasi from the University of Washington in St. Louis

- Allan Knopka from Pacific Northwest National Laboratory.

In his presentation, “Constructing a Predictive Model of Microbial Behavior”, Baliga discussed using biological questions to drive an iterative, mutidisciplinary systems research approach and the ultimate systems biology goal of predictive models. Baliga closely studies halophilic archaea such as Halobacterium salinarium NRC-1, which are extremophiles that thrive in areas with high levels of salt.

Pakrasi, a Lead Investigator for EMSL’s Membrane Biology Grand Challenge, discussed his work with cyanobacterial membrane systems, highlighting the benefit systems biology tools at EMSL have offered his research. Cyanobacteria have a robust circadian lifestyle, performing photosynthesis during the day and nitrogen fixation at night. The ultimate goal of Pakrasi's research is to engineer microbes with enhanced clean energy production abilities.

Knopka's presentation, Advanced technology for microbial communities, focused on the transforming technologies in the area of microbial communities research, including stable isotope-based technologies, methods to analyze heterogeneity in microbial populations, as well as tools for high-throughput and efficient cultivation.

Steven Wiley, EMSL lead scientist in biology, explained how SMERF is part of EMSL's efforts to continually evolve to best serve the needs of EMSL users. EMSL's Recapitalization Plan offers an opportunity for the scientific community to provide guidance as to how the recapitalization effort should be used to best further the needs of systems microbiology. EMSL already houses many capabilities relevant to systems microbiology, including:

- $\quad$ NMR and EPR, including the NMR/confocal capability

- Mass spectrometry, including AMT, PRISM, and community profiling tools

- Imaging and spectroscopy capabilities, including STORM microscopy for ultra-high resolution microbial imaging

- Prototype capabilities for metabolism studies, including an NMR-bioreactor to monitor microbial metabolism and LN-NMR for metabolomics

- Prototype molecular research techniques, including TAP-tag protein-protein interaction mapping and laser-capture microdissection of microbial biofilms

- Prototype bioinformatics capabilities, including an integrated bioinformatics software suite.

\section{Challenges: Single cells vs. populations}

Relating components to cells (molecular properties of components and how they interact in cells) is an area of research that is moving quickly, but bridging the gap between elegant, "simple" single cell systems and communities is complicated. Studies must be geared to the scope of communities and fundamental questions must be addressed such as: What are the fundamental units in a community? How do these units vary as a function of the species? 
It is also imperative to define the limits and nature of different experimental modalities and when it is appropriate to apply them. For example, scientists must determine when it is appropriate to look at individual cells versus the cell population. It is also important to know what level of granularity is necessary to predict what's happening at different levels (e.g., the species level, population level, and individual-cell level).

\section{Benefits and challenges of microbial studies}

Studying microbes comes with inherent benefits and challenges. On a positive note, all microbes have a high biomass, which is appealing for high-throughput studies. In addition, extremophiles are relatively simplistic compared to conventional bacteria (consider a salt mine population compared to soil population) and slow growing, offering a long time span to study transcription and translation.

Their limited flexibility also offers advantages. With only $1-2 \%$ of the genome given to regulation (compared to $10 \%$ in other bacteria), their regulatory repertoire is constrained. Therefore, they have simpler networks to infer - an advantage for scaling up from the cell to community level. Studies have shown that microbes do not respond independently to environmental variables; they have binned their responses into an evolutionary pattern. This same simplicity might be expected from communities.

However, bacteria are, in general, challenging to grow in the laboratory, and extremophiles are no exception. A subset might grow easily, but many others are challenging.

\section{Natural vs. artificial bacterial communities}

There is arguable value to using artificial bacterial communities - they are valuable as a model for cell-cell interactions and for understanding basic principles. Off-the-shelf organisms can also be used for mutation studies. However, artificial systems have their limitations.

Though difficult to grow, it is important to study natural cultures, to understand community function with respect to DOE needs, and to understand a community in a 3-D structure rather than liquid culture. Having the goal of growing difficult-to-culture microbes is good, but specific capabilities are needed to do this. An estimated $50 \%$ is the highest rate of success that can be reasonably expected.

\section{Sample preparation needs and concerns}

Sample preparation includes all of the steps needed from cell culture, collection, and storage to processing samples for analysis. Sample preparation also includes cell isolation for sub-culturing. Straightforward needs for sample preparation include automatic sampling and anaerobic sample processing. The latter capability will enable researchers to preserve the state of cells, to separate cells if necessary, and to rapidly freeze samples. In addition, anaerobic sample processing may be desirable for proteomics/metabolomics and is needed to study changes in redox. Laser capture capability is also desired; it is a preferred method to biofilm dissection because a cut across a biofilm yields a heterogeneous sample.

Note that hydrogen sulfide-producing organisms are a concern because of probe contamination and potential toxic gas production. Users and their microbe of study need to be screened and classified carefully and monitored accordingly.

\section{Cell growth and monitoring}

Cell growth and monitoring covers the growth of biomass under controlled conditions for 
generating analytical samples as well as for evaluating their physiological state. A variety of tools is needed for cell growth and monitoring.

\section{Parallel cultures, chemostats, and sampling}

A unique facility should not focus on batch culture. Rather, a focus on parallel micro-cultures, (not to exceed hundreds of cultures), is probably best. EMSL's new culture capability should be purchased off-the-shelf and modified for the types of experiments that are envisioned to be performed within the next five years.

Of the two micro-culture systems available - microfluidics with built-in chemostats and microreactors - chemostat cultures are better, even given their sampling volume constraint. (Note that though microreactors can be scaled to hundreds of milliliters, they may not be easily purchased off-the-shelf). Microfluidics w/ built-in chemostats are helpful for time-course analysis, they can run in batch or continuous mode, and can be used for dynamic gene expression studies. The culture system chosen for the SMERF should allow:

- $\quad$ Simultaneous measurements

- $\quad$ Rapid (intervals of seconds) sequential analysis

- Constant monitoring

- Response monitoring

- Generation of milligrams of cells for 'omics measurements

- Optimization of culture conditions

With more cultures, the number of microarrays needed to monitor them will increase exponentially. However, microarrays are being phased out in favor of direct sequencing and are, therefore, not a heavy concern. The EMSL should consider investing in new sequencing technologies for monitoring gene expression profiles of microbes.

\section{Accommodating extremophiles, accommodating users}

A flexible facility will cater to many users, therefore, the ability to cultivate microbes that thrive under a variety of conditions (e.g., high salt, high temperature, high pressure, high acidity, radioactive, and metallic) is needed. In addition, it would be useful to replicate environmental conditions, including atmospheric conditions and soil habitats as well as low-temperature, highlatitude, and methane environments.

Autoclave-level bioreactors are expected to be difficult to implement, but should have very high user demand. They replicate the high-temperature, high-pressure conditions of oil fields and are too expensive for individual labs. Additional feedback is needed to determine cultivation needs for virus-control of populations. Field-deployable, portable technology is a future focus; for example, technology that could be used to measure Geobacter proteomics onsite in groundwater.

The challenges of growing parallel cultures include monitoring and optimization. Monitoring each bioreactor of a massively parallel system generates massively parallel data because each bioreactor can be modified to have particular conditions (e.g., temp, light, $\mathrm{O}_{2}$, and salinity). Therefore, users must be offered data analysis expertise. In addition, each strain, condition, and variable will change the growth characteristics and require optimization; chemostats must be used to control the growth rates. For example, with ten strains and 100 reactors, each group will reach the stage at which stress response measurements must be taken at different times. Being able to program different sampling times for each culture is needed as is the ability to sample each culture "at will". 


\section{Accommodating photosynthetic microbes}

The SMERF should accommodate photosynthetic microbes, such that many light parameters can be controlled (e.g., intensity, wavelength, time synchronization, etc.). In addition, SMERF's photo-bioreactor design should address illumination homogeneity. Top-down light is not suitable; it only gives light to the top of the culture, making it difficult to quantify the amount of light each organism receives. There are some published designs for photosynthetic culture systems (e.g., Bernhard Palsson). Making these advanced photo-bioreactors widely available to the community would be extremely useful.

Note that high-intensity/low-heat-producing light sources are becoming affordable in large quantities; their on/off time is short. Note that gas exchange in a photo-bioreactors is an issue $\left(\mathrm{CO}_{2}\right.$ dissolution and stripping of $\left.\mathrm{O}_{2}\right)$. In addition, there is an explosion of interest in photobioreactors because of their biofuel link (e.g., oils from algae).

Photo-bioreactorcapability must accommodate:

- Light cycle and gas control

- Niche partitioning

- Light quality

- Variation in $\mathrm{CO}_{2}$ sequestration

- $\quad$ Light sensors to measure illumination intensity.

\section{Biofilms}

Biofilms are very relevant to DOE mission areas. The EMSL facility needs to complement, but not replicate, the biofilm facility at Montana State University. In regard to biofilms, SMERF should address high-throughput data capture and management, biofilms heterogeneity, and intrabiofilm microbial interactions.

There are no good commercial systems available for high-throughput, multi-measurement biofilms capabilities, but EMSL could potential develop something to meet this need. The physiology of biofilms is heterogeneous; therefore, measuring their spatial physiology is difficult. Several different approaches could be taken to overcome this challenge. For example, technology could be assembled to measure the proteome of biofilm cells located close to the surface as well as deep within the structure. SMERF should provide the ability to look at a variety of surfaces (e.g., oxides) and how they interact with biofilms. The surface science capabilities within EMSL should be used to complement SMERF needs in this way. Data management for SMERF is imperative; the kinds of data to be gathered from biofilm studies needs to be determined early and be appropriate for modeling these complex communities.

\section{Microbial communities}

To translate organism studies to a higher level, it is necessary to know its relevance to the community, but the community concept must be defined and organism/community data properly interpreted. Communities cultivated ex situ are not as desirable as in situ studies. To design appropriate culture facilities for microbial communities first required defining the relevant questions to be asked and the organisms that will be used to answer those questions.

\section{Analysis}

Analysis includes compositional analysis as well as measurement of gene expression and physiologically important parameters. This can be done at both the single-cell as well as population level; live-cell and dynamic measurements are also included. New types of analyses should be developed for SMERF, with the caveat that some technologies will be more useful than others for answering specific biological questions. 


\section{Proteomics and flow cytometry studies}

The number of samples generated by a parallel culture system will necessitate the use of high throughput technologies that have both great sensitivity and dynamic range. Post-translational proteomics, global proteomics, and combined metagenomics-proteomics are all very important technologies that SMERF should facilitate.

The complexity of connecting single-cell data to the community (and vice versa) can be reduced by using sequenced organisms. In addition, separating members of the community by using flow cytometry should allow powerful proteomics studies to be conducted on small groups of cells. Flow cytometry could greatly enhance the usefulness of the SMERF, especially if instruments optimized for aerobic and anaerobic microbes were employed. This would be a good area to collaborate with industry.

\section{Metabolomics and fluxomics}

Metabolomics is imperative, but the path forward for metabolomics is not as clear as that for proteomics. Identifying unknowns is a difficult path, which requires synthesis of standards and employing an organic chemist. It is far more difficult to identify an unknown metabolite than an unknown protein.

A metabolomics approach is needed to identify unknown metabolites, quantify certain set of specific metabolites, and measure the flux of the metabolites. Each set of information provides necessary information is understanding the metabolic pathways and capabilities of a given organism. For example, delineating specific metabolic pathways is not possible with flux measurements alone. Both metabolite identification and flux measurements are critical for understanding these pathways.

The vast majority of metabolites that are likely to be observed, however, are not unique and do not change their concentrations over time. It appears likely that cells have evolved to keep them constant. Therefore, targeted metabolomics (also called metabolic profiling or the identification of signature metabolites) is more efficient than whole-cell metabolomics and likely to be quite informative. Much information can be obtained from studies of major metabolites, such as ATP, ADP, NADH, NADPH. Remarkably few metabolites are needed to understand most cellular metabolic pathways.

Note that measuring the dynamics of isotopically-labeled metabolite precursors is a good approach for metabolite flux studies.

\section{Imaging}

For network and community studies, spatial resolution studies are important - by examining the distribution and activities of individual cells, much can be learned about communities. Facilities for in situ studies should be able to accommodate artificial and natural communities (from the field or controlled cultures). In addition, some systems are intrinsically suited to high-throughput imaging studies, such as photosynthetic organisms with natural chlorophyll dyes.

Technologies/capabilities that would move microbiology imaging studies forward are:

- FiSH-probes

- Spatial distribution of microbes and their substructures (e.g., on mineral surfaces)

- High-speed, multi-spectral imaging (to look at reporter groups). Although multi-spectral imaging is an off-the-shelf capability, EMSL could modify the equipment to offer high speed

- High-resolution imaging in hydrated samples, using markers to look at distribution of proteins and other structures 
- Microspectroscopy

- Mapping of proteins within cells

- Raman spectroscopy to distinguish different organisms

- Maldi-ToF to image protein and metabolite distribution

\section{Data management and integration}

Data management and integration systems should consist of a collection of software and hardware tools that can collect, store and retrieve analytical data as well as the metadata needed to relate this data to the cell state. This category should also include specialized software needed to analyze data generated from any novel technologies or to integrate that data with other information.

\section{Data collection}

Metadata collected during an experiment (e.g., growth parameters) is crucial for interpreting high-throughput data and so this should be considered a fundamental data type to capture. In addition, users should be able to monitor the cultivation process online and provide feedback. For example, users should be able to modify growth parameters and trigger sample collection. This capability can be modeled after the current NMR system. Experience in EMSL with remotecontrolled microscopes should also prove useful for implementing this capability.

\section{Data management and analysis}

EMSL and PNNL already offer tools to store large datasets. For example, the Membrane Biology Grand Challenge project used PRISM and Sharepoint. The Environmental Biomarkers Initiative used a data management system connected to the Molecular Science Computing Facility's NWfs storage system. The Biomolecular Systems Initiative developed an Integrated Database for Experimental Analysis (IDEA). These efforts should be used as a starting point for data management for SMERF. All analysis tools should be able to access these databases and made freely available. Some algorithm development and expertise will likely be necessary.

Users should have access to tools that facilitates the handling and interpretation of highthroughput data sets. It is also critical that staff be available as consultants for data integration and interpretation. The Joint Genomes Institute model for data handling could be used and improved upon.

\section{Core database}

A core database on model organisms that allows the microbiology community to access 'omics data would be desirable to the scientific user community. These data, however, would need to be normalized in some way to allow them to be compared and analyzed with respect to differing parameters, such as culture conditions, etc.

An open data policy is necessary, but the details would have to be carefully worked out. Such a policy would state that data gathered using EMSL resources must be made available. This would allow the community to use these data for many types of analysis. The proposed DOE "knowledge base" may address some of these needs. For such a database to work, staff from multiple disciplines (e.g., microbiologists, network inference experts, bioinformaticists, etc.) must communicate openly from the beginning of the process. If data is not collected properly from the beginning, certain types of analyses will be impossible.

\section{Network inference and parallelization}

Data clustering can be parallelized as can some parts of network inference algorithms, but some data must be serially processed. Parallelization is necessary to process array data and to greatly 
speed up searches where a community of biologists are searching against large datasets, such as large sets of sequence data. EMSL and PNNL have the expertise needed for code parallelization, with ScalaBLAST being an example of a parallel program written at PNNL.

Appropriate experimental design must be used to be able to successfully infer biological networks from high-throughput data sets. A database of inferred networks would be valuable to be able to understand how cells function. However, maintaining and evolving databases is a challenge that must be addressed in building a SMERF. Perhaps it would be better to build smaller, connected databases rather than a single database for all of the data generated from the SMERF. 


\section{Appendix B: Report from a Workshop on Experimental Access Portals for a Global Proteomics Facility}

Gaithersburg, Maryland, June 2-3, 2003

Facilitator: H. Steven Wiley, Pacific Northwest National Laboratory

\section{Workshop Background and Purpose}

This workshop was sponsored by Pacific Northwest National Laboratory (PNNL) to address the question of scientific-community access to large-scale facilities such as those being planned by the U.S. Department of Energy (DOE)'s Genomes to Life (GTL) program. More than 40 scientists from academia, scientific institutions and agencies, and national laboratories attended. The workshop highlighted several issues of user interaction. In the context of this report, users are the broad microbiology scientific community.

The workshop's primary purpose was to define ways in which a community of scientists could potentially interact with a large core facility. As biology makes the transition to larger-scale, more multidisciplinary research projects, the importance of centralized resources is expected to increase. How these resources can be used most effectively to drive biological research is unclear. Biologists could change the way they do experiments, core facilities could be designed to integrate into the current workflow, or some mixture of the two approaches might occur.

The workshop was intended to examine the concept of "experimental access portals" (EAPs), which are integrated facilities where experimental scientists would prepare quality-controlled samples that would then be processed by separate high-throughput analytical resources. The EAP concept was presented as only one example of several possible ways by which biologists could interact with large-scale core facilities. By focusing on a specific initial concept as a starting point, it was felt that the participants could more productively discuss different ways to interface the work of individual scientists with large-scale resources.

\section{Workshop Overview}

The workshop consisted of a few targeted presentations followed by breakout sessions and group discussions. First was an overview of GTL facility plans and a discussion of PNNL's current ideas for a facility for whole proteome analysis and ideas regarding EAPs. The next three presentations outlined specific experimental scenarios that would use global proteomics or metabolomics. They detailed steps in the experimental "pipeline," points at which data and samples could be handed off to a core facility, and the logistics involved. Two presentations followed on how different types of data could be integrated and how communities of scientists could be engaged productively.

\section{Key Needs and Issues}

The workshop identified several key issues. First and paramount is the need for user education at two levels:

1. How large-scale facilities can drive scientific progress in the users' fields.

2. How to use tools, data types, and associated dynamic information to gain a higher-level understanding of important scientific questions.

A paradigm shift is involved in making the transition from small-scale to large-scale science, and education and training are needed to facilitate this evolution of biology. Community involvement is required so that scientific resources represented by large-scale facilities can be used to solve problems of the greatest significance and importance to the scientific community. 
A particular concern expressed in the workshop was the lack of understanding about how bioinformatics and computational biology can facilitate current research. Particular attention needs to be given to this area of user awareness.

The disparity between current and potential research modes that could drive progress in the GTL program complicates efforts to design an appropriate user interface or mechanism for user access to the facility. As biology moves from small scale to large scale, requirements for user access also will change and evolve. Nevertheless, several models of user interaction seem suitable for different scales of biological research.

\section{Models Recommended for Access to a Core Facility}

Three models were recommended:

1. For access by an individual investigator level, resources should allow research projects to be both initiated and completed at the core facility. In this model, general-purpose research labs for experiments and sample preparations should be an intrinsic part of the core facility.

2. For groups of investigators or research consortia with significant research resources and bioinformatics infrastructure, only selected parts of a project would need to be done in the core. This model would require a standardized way to exchange data and information between the two groups.

3. Large institutions, such as universities or research institutes could be directly involved in the planning and execution of research in the core facility. Workshop consensus was that several linked, but separate experimental facilities could serve very effectively as sources of large volumes of high-quality samples as well as centers of data interpretation and integration.

The systems biology revolution is in the early stages, so there is currently little community demand for the facilities needed for large-scale biology projects. Workshop participants, however, expressed a clear appreciation of the power of this approach to solve important problems. No one overarching technical problem related to systems biology is apparent in the biological community, so the facility cannot be built with specifications aimed toward addressing such a problem. Specifying a class or classes of problems to be solved using a systems approach will be just as critical in facility design as technology and instrumentation. Coupling a series of prototype problems with a prototype facility is likely to be an effective strategy for designing a successful facility.

\section{Summary Discussions}

Following each set of presentations, workshop participants had the chance to provide feedback. This section summarizes their discussions, with comments grouped into Critical Issues and Recommendations.

\section{Critical Issues}

- The stated mission of GTL facilities is that they will enable systems biology and the understanding of microbes. This needs more definition. The science to be done in these facilities should be stated precisely to justify their specific configurations and indicate the user community that will be involved.

- An experimental database must be at the heart of this facility and must support modeling and simulation. The database also must be able to check for bad samples and data. It must be structured to allow evaluation of sample quality while still allowing one-time experiments. 
- When implementing a distributed data resource, establishing who gets research credit is important. Typically, an author is responsible for everything in a paper, which is difficult to ensure when dozens of people are involved in the experiment. This kind of science means that a new definition is needed for what constitutes an author.

- Developing data-release policies and establishing rules governing access to archived data will be important.

- Establishing how to use this new style of experimental modality, in which simulation and large-scale experimentation are combined to focus on a specific scientific question, is important. Prototype goal-oriented problems are needed to serve as examples to other scientists.

- In the case of large, multi-investigator experimental studies, some data will be produced remotely and some at the facility. How data will be merged with metadata must be established.

- Establishing strict rules for sample chain of custody is essential. The number of samples will be very large, making problems associated with sample mix-ups that much greater. Welldefined strains of organisms must be used. They must be the same as the sequenced organisms and must have some way to verify their identities. The expense of large-scale experiments requires a much higher standard.

\section{Recommendations}

- Accountability must be clear for production as well as experimental design and execution.

- Exploring cross-agency interactions to build the program will be useful.

- A high priority should be placed on bringing innovation into the groups. Connect big science with little science within universities.

- The science mix must be balanced. We don't yet know what types of science will benefit most from a high-throughput approach, so we should explore various questions early in the program.

- All critical issues with respect to the user community are issues of engagement. Outline these issues and give potential solutions to them (e.g., how individual investigators can compete with industry if they don't have access to these large facilities). User-access portals appear to be a useful way to address this issue.

- All data should be placed into a knowledge base that can be accessed by everyone, and comprehensive modeling, analysis, and computational tools should be offered.

- Facilities also should provide standardized cultures for projects and associated users in the community.

\section{Overall Conclusions}

The potential of large-scale biological resources is evident but primarily from a theoretical rather than a practical viewpoint. Very few examples of successful large-scale projects can serve as reasonable paradigms for the class of problems GTL was designed to address. Thus, vigorously engaging the user community through education, training, and pilot projects is essential to build awareness and advocacy for this approach to biology.

There is not a single overarching problem with respect to microbial systems biology, so a facility cannot be built with specifications aimed toward solving a single problem. However, using a prototype problem to design a prototype facility is likely to be necessary for success. 


\section{Participants}

June 2-3, 2003, Gaithersburg, MD

Eric Ackerman

Pacific Northwest National Laboratory

eric.ackerman@pnl.gov

Carl Anderson

Brookhaven National Laboratory

cwa@bnl.gov

Joe Beckman

The Linus Pauling Institute

joe.beckman@oregonstate.edu

Jim Bixler

Pacific Northwest National Laboratory

jim.bixler@pnl.gov

Fred Brockman

Pacific Northwest National Laboratory

fred.brockman@pnl.gov

Cory Bystrom

Oregon Health Sciences University

bystrom@ohsu.edu

Bill Costerton

Montana State University

bill_c@erc.montana.edu

Mike Daly

University of the Health Sciences

mdaly@usuhs.mil

Daryll DeWald

Utah State University

dewald@biology.usu.edu

George Dylan

National Science Foundation

dgeorge@nsf.gov

Norm Dovichi

University of Washington

dovichi@chem.washington.edu

Leland Ellis

U. S. Department of Agriculture

lce@ars.usda.gov
Ted Einstein

University of Maryland-MRSEC

einstein@physics.umd.edu

Marvin Frazier

U.S. Department of Energy

Life Sciences Division

MARVIN.FRAZIER@science.doe.gov

Paul Freimuth

Brookhaven National Laboratory

freimuth@bnl.gov

Julie Gephart

Pacific Northwest National Laboratory

julie.gephart@pnl.gov

Athula Herath

Oxford GlycoSciences (U.K.)

Athula.Herath@ogs.co.uk

Rick Horwitz

University of Virginia School of Medicine

Horwitz@virginia.edu

Trey Ideker

Whitehead Institute for Biomedical

Research

trey@wi.mit.edu

Michael Kahn

Washington State University

kahn@wsu.edu

Sam Kaplan

University of Texas

Samuel.Kaplan@uth.tmc.edu

Jay Keasling

Lawrence Berkeley National Laboratory

jdkeasling@lbl.gov

Mike Knotek

DOE Consultant

m.knotek@verizon.net 
Frank Larimer

Oak Ridge National Laboratory

larimerfw@ornl.gov

Mary Lipton

Pacific Northwest National Laboratory

mary.lipton@pnl.gov

Peter Lyster

National Institutes of Health

Lysterp@mail.nih.gov

Dennis Maeder

University of Maryland-Biotechnology

Institute

maeder@umbi.umd.edu

Betty Mansfield

Oak Ridge National Laboratory

mansfieldbk@ornl.gov

Cheryl Marks

National Cancer Institute

marksc@mail.nih.gov

Blaine Metting

Pacific Northwest National Laboratory

blaine.metting@pnl.gov

George Michaels

Pacific Northwest National Laboratory

george.michaels@pnl.gov

Matthew Parsek

Northwestern University

m-parsek@northwestern.edu

Orna Resnekov

The Molecular Sciences Institute

Orna.resnekov@molsci.org

Frank Robb

University of Maryland-Biotechnology

Institute

robb@umbi.umd.edu

Karin Rodland

Pacific Northwest National Laboratory karin.rodland@pnl.gov

Karen Skinner

National Institutes of Health

kskinner@ngmsmtp.nida.nih.gov

Tom Squier

Pacific Northwest National Laboratory

tom.squier@pnl.gov

Sudhir Srivastava

National Cancer Institute

srivasts@mail.nih.gov

Mary Stenzel-Poore

Oregon Health Sciences University

poorem@ohsu.edu

Marietta Strachan

Pacific Northwest National Laboratory

marietta.strachan@pnl.gov

Michael Teresinski

U.S. Department of Energy

Life Sciences Division

michael.teresinski@science.doe.gov

David Thomassen

U.S. Department of Energy

Life Sciences Division

David.Thomassen@science.doe.gov

Brian Thrall

Pacific Northwest National Laboratory

brian.thrall@pnl.gov

Ed Uberbacher

Oak Ridge National Laboratory

uberbacherec@ornl.gov

Bart Weimer

Utah State University

bcweimer@cc.usu.edu

Steven Wiley

Pacific Northwest National Laboratory

steven.wiley@pnl.gov 